\title{
ASYMPTOTICALLY MOST POWERFUL RANK-ORDER TESTS ${ }^{1}$
}

\section{By JarosLav HÁJEK}

\section{Czechoslovak Academy of Sciences and University of California, Berkeley}

0. Summary. Having observed $X_{i}=\alpha+\beta c_{i}+\sigma Y_{i}$, we test the hypothesis $\beta=0$ against the alternative $\beta>0$. We suppose that the square root of the probability density $f(x)$ of the residuals $Y_{i}$ possesses a quadratically integrable derivative and define a class of rank order tests, which are asymptotically most powerful for given $f$. The main result is exposed in the following succession: theorem, corollaries and examples, comments, preliminaries and proof. The proof is based on results by Hájek [6] and LeCam [8], [9]. Section 6 deals with asymptotic efficiency of rank-order tests, which is shown, on the basis of Mikulski's results [10], to be presumably never less than the asymptotic efficiency of corresponding parametric tests of Neyman's type [11]. This would extend the wellknown result obtained by Chernoff and Savage [2] for the Student $t$-test. Furthermore, it is shown that the efficiency may be negative, i.e., asymptotic power may be less than the asymptotic size. In Section 7 we consider parallel rank-order tests of symmetry for judging paired comparisons. Section 8 is devoted to rankorder tests for densities such that $(f(x))^{\frac{1}{2}}$ does not possess a quadratically integrable derivative. In Section 9, we construct a test which is asymptotically most powerful simultaneously for all densities $f(x)$ such that $(f(x))^{\frac{1}{2}}$ possesses a quadratically integrable derivative.

1. The main theorem. Consider a sequence of random vectors $\left(X_{\nu 1}, \cdots, X_{\nu N_{\nu}}\right), 1 \leqq \nu<\infty$, where the $X$ 's are independent and

$$
P\left(X_{\nu i} \leqq x \mid \alpha, \beta, \sigma\right)=F\left(\left(x-\alpha-\beta c_{\nu i}\right) / \sigma\right), \quad 1 \leqq i \leqq N_{\nu}, 1 \leqq \nu<\infty,
$$

where $F$ is a known distribution function, $c_{\nu i}$ are known constants, $\alpha$ and $\sigma$ are nuisance parameters, $-\infty<\alpha<\infty, \sigma>0$, and $\beta$ is the parameter under test. We test the hypothesis $\beta=0$ against the alternative $\beta>0$.

Assume that the density $f(x)=F^{\prime}(x)$ exists and that $(f(x))^{\frac{1}{2}}$ possesses a quadratically integrable derivative. As

$$
\left((f(x))^{\frac{1}{2}}\right)^{\prime}=\frac{1}{2}\left[f^{\prime}(x) /(f(x))^{\frac{1}{2}}\right]
$$

it means that

$$
\int_{-\infty}^{\infty}\left[f^{\prime}(x) / f(x)\right]^{2} f(x) d x<\infty .
$$

Let the constants $c_{\nu i}$ satisfy the Noether condition

$$
\lim _{\nu \rightarrow \infty}\left\{\left[\max _{1 \leqq i \leqq N_{\nu}}\left(c_{\nu i}-\bar{c}_{\nu}\right)^{2}\right] /\left[\sum_{i=1}^{N_{\nu}}\left(c_{\nu i}-\bar{c}_{\nu}\right)^{2}\right]\right\}=0
$$

Received May 19, 1961; revised April 23, 1962.

${ }_{1}$ Prepared with the partial support of the National Science Foundation, Grant G-14648. 
where $\bar{c}_{\nu}=N_{\nu}^{-1} \sum_{i=1}^{N_{\nu}} c_{\nu i}$, and the boundedness condition

$$
\sup _{\nu} \sum_{i=1}^{N_{\nu}}\left(c_{\nu i}-\bar{c}_{\nu}\right)^{2}<\infty .
$$

Denoting the inverse of $F$ by $F^{-1}$, let us introduce the following function:

$$
\varphi(u)=-\left[f^{\prime}\left(F^{-1}(u)\right) / f\left(F^{-1}(u)\right)\right], \quad 0<u<1 .
$$

From (1.3) is follows that

$$
\int_{0}^{1} \varphi(u) d u=\int_{-\infty}^{\infty} f^{\prime}(x) d x=0
$$

and

$$
\int_{0}^{1} \varphi^{2}(u) d u=\int_{-\infty}^{\infty}\left[f^{\prime}(x) / f(x)\right]^{2} f(x) d x<\infty .
$$

If to an $f$ there corresponds $\varphi, f \rightarrow \varphi$, then to $(1 / \sigma) f((x-\alpha) / \sigma)$ there corresponds $(1 / \sigma) \varphi(u)$ :

$$
(1 / \sigma) f((x-\alpha) / \sigma) \rightarrow(1 / \sigma) \varphi(u),
$$

i.e., the map is invariant under translation and a change of scale only introduces a multiplicative constant.

We shall need the following function and constants:

$$
\begin{gathered}
\Phi(x)=(2 \pi)^{-\frac{1}{2}} \int_{-\infty}^{x} \exp \left(-\frac{1}{2} t^{2}\right) d t \\
\Phi\left(K_{\epsilon}\right)=1-\epsilon, \\
d_{\nu}^{2}=\sum_{i=1}^{N_{\nu}}\left(c_{\nu i}-\bar{c}_{\nu}\right)^{2} \int_{-\infty}^{\infty}\left[f^{\prime}(x) / f(x)\right]^{2} f(x) d x .
\end{gathered}
$$

Now let $R_{\nu i}$ be the rank of $X_{\nu i}$ in the ordered sample $V_{\nu 1}<\cdots<V_{\nu N_{\nu}}$, i.e.,

$$
X_{\nu i}=V_{\nu_{R_{\nu}}}, \quad 1 \leqq i \leqq N_{\nu}, 1 \leqq \nu<\infty .
$$

Furthermore, let $\varphi_{\nu}(u)$ be a function defined on $(0,1)$ and constant over intervals $\left(i / N_{\nu},(i+1) / N_{\nu}\right), 0 \leqq i<N_{\nu}$, i.e.,

$$
\varphi_{\nu}(u)=\varphi_{\nu}\left(i /\left(N_{\nu}+1\right)\right), \quad(i-1) / N_{\nu}<u<i / N_{\nu} .
$$

Consider rank-order statistics $S_{\nu}$ expressible in the following form:

$$
S_{\nu}=\sum_{i=1}^{N_{\nu}}\left(c_{\nu i}-\bar{c}_{\nu}\right) \varphi_{\nu}\left(R_{\nu i} /\left(N_{\nu}+1\right)\right), \quad 1 \leqq \nu<\infty .
$$

Let $\epsilon_{\nu}$ and $Q_{\nu}(\beta, \sigma)$ be the size and power of the test based on critical region

$$
S_{\nu}>K_{\epsilon} d_{\nu}
$$

$1 \leqq \nu<\infty$,

where $K_{\epsilon}$ and $d_{\nu}$ are given by (1.11) and (1.12). 
Definition 1.1. If $\lim _{p \rightarrow \infty} \epsilon_{\nu}=\epsilon$ and

(1.17) $\lim \inf _{\nu \rightarrow \infty}\left[Q_{\nu}(\beta, \sigma)-Q_{\nu}^{*}(\alpha, \beta, \sigma)\right] \geqq 0, \quad-\infty<\alpha<\infty, \beta>0, \sigma>0$, where $Q_{\nu}^{*}(\beta, \sigma)$ is the power of any other test of limiting size $\epsilon$, we say that the test based on (1.15) is asymptotically uniformly most powerful (see Neyman [11]). Of course, uniformness is meant with respect to $\alpha, \beta$ and $\sigma$.

Theorem 1.1. Consider model (1.1) and suppose that conditions (1.3), (1.4) and (1.5) are satisfied. Let

$$
\lim _{\nu \rightarrow \infty} \int_{0}^{1}\left[\varphi_{\nu}(u)-\varphi(u)\right]^{2} d u=0,
$$

where $\varphi$ is given by (1.6). Then the critical region (1.16) provides an asymptotically uniformly most powerful test of limiting size $\epsilon$, and the asymptotic power equals

$$
1-\Phi\left(K_{\epsilon}-(\beta / \sigma) d_{\nu}\right),
$$

where $\Phi, K_{\epsilon}$ and $d_{\nu}$ are given by (1.10), (1.11) and (1.12).

2. Corollaries and examples. Step-functions $\varphi_{\nu}$ form an $N_{\nu}$-dimensional subspace in the space of quadratically integrable functions on $(0,1)$. The projection of $\varphi$ on this subspace, say $\varphi_{\nu}^{0}$, equals

$$
\varphi_{\nu}^{0}\left(i /\left(N_{\nu}+1\right)\right)=N_{\nu} \int_{(i-1) / N_{\nu}}^{i / N_{\nu}} \varphi(u) d u, \quad 1 \leqq i \leqq N_{\nu} .
$$

If we extend the definition of $\varphi_{\nu}^{0}$ to the whole interval $(0,1)$ according to $(1.14)$, we easily see that (1.19) is satisfied.

Let $V_{\nu 1}<\cdots<V_{\nu N_{\nu}}$ be the ordered sample referring to $F$ and $\alpha=\beta=0$ and $\sigma=1$, so that $Z_{\nu 1}<\cdots<Z_{\nu N_{\nu}}$, where $Z_{\nu i}=F\left(V_{\nu i}\right)$, is an ordered sample from the uniform distribution over $(0,1)$. Put

$$
\varphi_{\nu}^{+}\left(i /\left(N_{\nu}+1\right)\right)=E\left[-\left(f^{\prime}\left(V_{\nu i}\right) / f\left(V_{\nu i}\right)\right)\right]=E\left[\varphi\left(Z_{\nu i}\right)\right], \quad 1 \leqq i \leqq N_{\nu},
$$

and complete the definition of $\varphi_{\nu}$ according to (1.14). Then, on account of Lemma 6.1 of [6], $\varphi_{\nu}^{+}$satisfies the condition (1.19), too. So we get

Corollary 2.1. Let the conditions (1.3), (1.4) and (1.5) be satisfied. Put

$$
S_{\nu}^{0}=\sum_{i=1}^{N_{\nu}}\left(c_{\nu i}-\bar{c}_{\nu}\right) \varphi_{\nu}^{0}\left(R_{\nu i} /\left(N_{\nu}+1\right)\right)
$$

and

$$
S_{\nu}^{+}=\sum_{i=1}^{N_{\nu}}\left(c_{\nu i}-\bar{c}_{\nu}\right) \varphi_{\nu}^{+}\left(R_{\nu i} /\left(N_{\nu}+1\right)\right),
$$

where $\varphi_{\nu}^{0}$ and $\varphi_{\nu}^{+}$are defined by (2.1) and (2.2). Then the critical regions $S_{\nu}^{0}>K_{\epsilon} d_{\nu}$ and $S_{\nu}^{+}>K_{\epsilon} d_{\nu}$ both provide an asymptotically most powerful test of limiting size $\epsilon$ and of asymptotic power (1.20).

Another step-function of particular interest is defined as follows:

$$
\varphi_{\nu}^{*}\left(i /\left(N_{\nu}+1\right)\right)=\varphi\left(i /\left(N_{\nu}+1\right)\right), \quad 1 \leqq i \leqq N_{\nu} .
$$


TABLE 1

\begin{tabular}{|c|c|c|c|}
\hline$\dot{0}$ & $f(x)$ & $-\frac{f^{\prime}(x)}{f(x)}$ & $\varphi(u)$ \\
\hline 1 & $\frac{1}{2} e^{-|x|}$ & $\operatorname{sign} x$ & $\operatorname{sign}\left(u-\frac{1}{2}\right)$ \\
\hline 2 & $\begin{array}{l}\sqrt{3} e^{-x \sqrt{3}} \\
\quad \cdot\left(1+e^{-x \sqrt{3}}\right)^{-2}\end{array}$ & $\sqrt{3}\left(1-e^{-x \sqrt{3}}\right)\left(1+e^{-x \sqrt{3})^{-1}}\right.$ & $2 \sqrt{3}\left(u-\frac{1}{2}\right)$ \\
\hline 3 & $(2 \pi)^{-\frac{1}{2}} \exp \left(-\frac{1}{2} x^{2}\right)$ & $x$ & $\Phi^{-1}(u)$ \\
\hline 4 & $\exp \left(x-e^{x}\right)$ & $e^{x}-1$ & $\begin{array}{r}-\log (1-u) \\
-1\end{array}$ \\
\hline 5 & $\begin{array}{l}\frac{1}{3}\left[\phi\left(\frac{10}{3} x\right)\right. \\
\left.\quad-\phi\left(\frac{10}{3} x-10\right)\right]\end{array}$ & $\frac{10}{3}(2 \pi)^{-\frac{1}{2}} \frac{\exp \left[-\frac{1}{2}\left({ }^{10} x-10\right)^{2}\right]-\exp \left[-\frac{1}{2}\left(\frac{10}{3} x\right)^{2}\right]}{\Phi\left(\frac{10}{3} x\right)-\Phi\left(\frac{10}{3} x-10\right)}$ & See Fig. 1 \\
\hline
\end{tabular}

Here, of course some continuity properties are needed. From Lemma 2.2 of [6] it follows that monotoneity of $\varphi$ is sufficient. Obviously, it also suffices, if $\varphi$ is continuous and bounded on $(0,1)$. So we get

Corollary 2.2. Let the conditions (1.3), (1.4) and (1.5) be satisfied. Put

$$
S_{\nu}^{*}=\sum_{i=1}^{N_{\nu}}\left(c_{\nu i}-\bar{c}_{\nu}\right) \varphi\left(R_{\nu i} /\left(N_{\nu}+1\right)\right)
$$

and suppose that $\varphi(u)$ is either monotoneous or continuous and bounded. Then the critical region $S_{\nu}^{*}>K_{\epsilon} d_{\nu}$ provides an asymptotically most powerful test of limiting size $\epsilon$ and of asymptotic power (1.20).

In Table 1 there are listed five various densities together with corresponding logarithmic derivatives and functions $\varphi(u)$. The functions $\varphi(u)$ are drawn on Fig. 1 (see page 1128).

The first density in Table 1 is called double exponential. The corresponding $S_{\nu}^{*}$-test may be called median test, because in the two-sample problem (when the $c_{v i}$ 's attain only two different values) it consists in counting the number of observations from the first sample, which are greater than the median of the pooled sample.

The logistic density is listed as the second one. The constant $\sqrt{3}$ has been introduced to get comparable curves in Fig. 1. All three tests based on $S_{\nu}^{0}, S_{\nu}^{+}$ and $S_{\nu}^{*}$, respectively, reduce to the Wilcoxon test [14].

The density No. 3 is normal. The $S_{\nu}^{+}$-test is called the Fisher-Yates-TerryHoeffding test and $S_{\nu}^{*}$-test is called van der Waerden's test [13].

The fourth density has been obtained by the transformation $y=e^{x}$ from the exponential density $e^{-y}, y \geqq 0$. So testing location changes of the former density may be reinterpreted as testing scale changes of the latter one. The $S_{\nu}^{+}$-test may be called the I. R. Savage test, who showed [12] that

$$
1+\varphi_{\nu}^{+}\left(i /\left(N_{\nu}+1\right)\right)=\sum_{j=N_{\nu}-i+1}^{N_{\nu}}(1 / j) .
$$




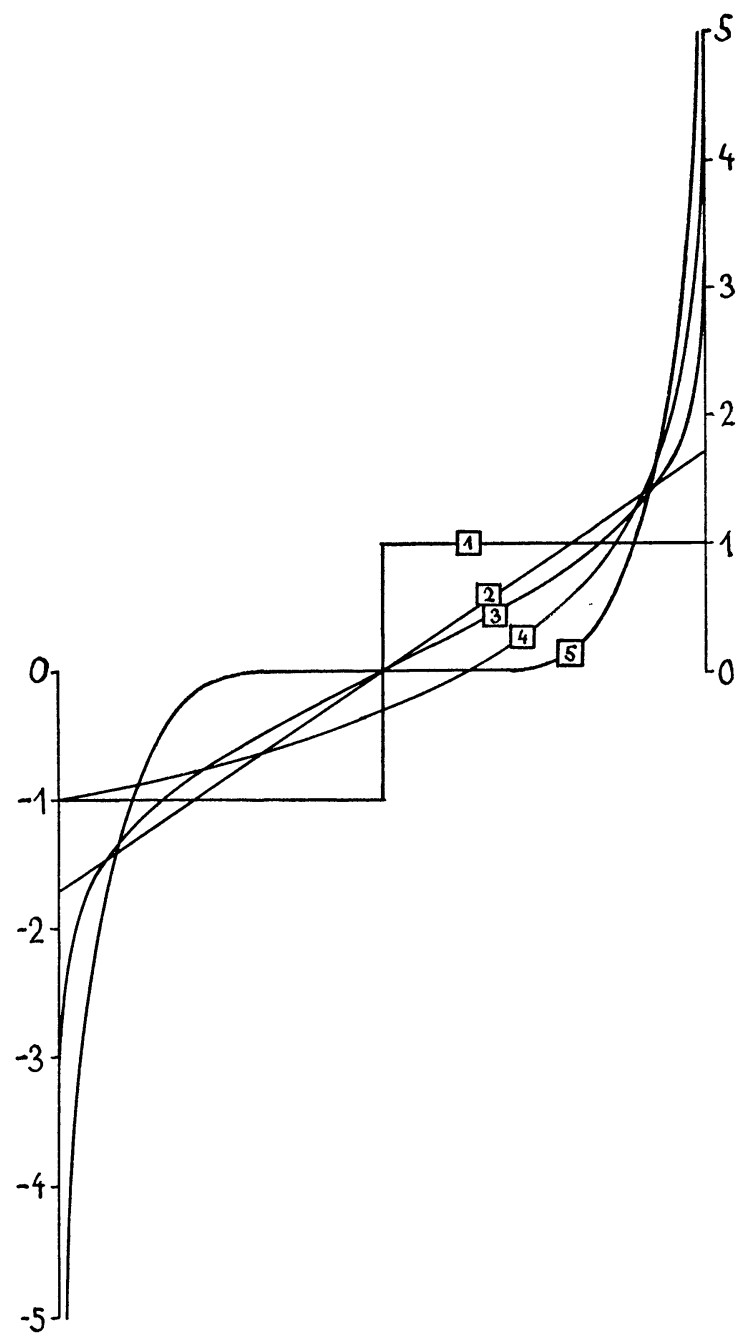

FIG. 1. The curves $\varphi(u)$ are numbered according to Table 1

The last distribution is a convolution of the normal distribution with zero mean and $\sigma=0,3$ and of the uniform distribution over the interval $(0,3)$. In this case the rank order tests emphasize the role of the tails of the ordered sample.

3. Some comments.

3.1. The two sample problem. If

$$
\begin{aligned}
c_{\nu i} & =0, & & 1 \leqq i \leqq n_{\nu}, \\
& =\Delta_{\nu}, & & n_{\nu}<i \leqq N_{\nu},
\end{aligned}
$$

we get the two sample problem as a special case of our regression model. As- 
sumptions (1.4) and (1.5) then reduce to

$$
\lim _{\nu \rightarrow \infty} \max \left\{n_{\nu}^{-1}-N_{\nu}^{-1},\left(N_{\nu}-n_{\nu}\right)^{-1}-N_{\nu}^{-1}\right\}=0
$$

and

$$
\sup _{\nu} n_{\nu} \Delta_{\nu}^{2}\left(1-\left(n_{\nu} / N_{\nu}\right)\right)<\infty
$$

respectively. Obviously, (3.2) is equivalent to the requirement that the sizes of the two samples, $n_{\nu}$ and $N_{\nu}-n_{\nu}$, both tend to $\infty$. Otherwise, however, they may be quite arbitrary. For example, the ratio $n_{\nu} / N_{\nu}$ may tend to 0 or 1 .

3.2. Nuisance parameters. Introducing the nuisance parameters $\alpha$ and $\sigma$ avoids some artificial assumption concerning the constants $c_{\nu i}$ and, furthermore, makes the knowledge of $F$ more plausible. If the distribution functions of $X_{\nu i}$ were $F\left(x ; \theta_{0}+\beta c_{\nu i}\right)$, we would have assumed that $\sum_{i=1}^{N_{\nu}} c_{\nu i}=0$, or at least

$$
N_{\nu} \bar{c}_{\nu}^{2}\left(\sum_{i=1}^{N_{\nu}} c_{\nu i}^{2}\right)^{-1} \rightarrow 0
$$

in order to get a situation, where asymptotically most powerful tests may be found among the rank-order tests. For this reason we have confined ourselves to a regression model, though the model with $P\left(X_{\nu i}<x\right)=F\left(x ; \theta_{0}+\beta c_{\nu i}\right)$ could be discussed with our methods, too.

3.3. We do not assume that $f(x)>0,-\infty<x<\infty$, so that individual admissible distributions may not be absolutely continuous with respect to each other.

3.4. The best parametric test statistic of Neyman's type [11] has the following simple form:

$$
M_{\nu}=-\sum_{i=1}^{N_{\nu}}\left(c_{\nu i}-\bar{c}_{\nu}\right) \frac{f^{\prime}\left(\frac{X_{\nu i}-\hat{\alpha}_{\nu}}{\hat{\sigma}_{\nu}}\right)}{f\left(\frac{X_{\nu i}-\hat{\alpha}_{\nu}}{\hat{\sigma}_{\nu}}\right)},
$$

where $\hat{\alpha}_{\nu}$ and $\hat{\sigma}_{\nu}$ are some proper estimates of $\alpha$ and $\sigma$. If the estimates $\hat{\alpha}$ and $\hat{\sigma}$ satisfy the invariance conditions

$$
\hat{\alpha}\left(b X_{\nu 1}+a, \cdots, b X_{\nu N_{\nu}}+a\right)=b \hat{\alpha}\left(X_{\nu 1}, \cdots, X_{\nu N_{\nu}}\right)+a
$$

and

$$
\hat{\sigma}\left(b X_{\nu 1}+a, \cdots, b X_{\nu N_{\nu}}+a\right)=b \hat{\sigma}\left(X_{\nu 1}, \cdots, X_{\nu N_{\nu}}\right),
$$

then the distribution of $M_{\nu}$ will be invariant with respect to $\alpha$ and $\sigma$. If, moreover,

$$
\lim _{\nu \rightarrow \infty} E\left[\frac{f^{\prime}\left(\frac{X_{\nu 1}-\hat{\alpha}}{\hat{\sigma}}\right)}{f\left(\frac{X_{\nu 1}-\hat{\alpha}}{\hat{\sigma}}\right)}-\frac{f^{\prime}\left(\frac{X_{\nu 1}-\alpha}{\sigma}\right)}{f\left(\frac{X_{\nu 1}-\alpha}{\sigma}\right)}\right]^{2}=0,
$$

where $E$ refers to $\alpha=\alpha, \beta=0, \sigma=\sigma$, then it may be shown, by methods of 
Section 5, that the critical region $M_{\nu}>K_{\epsilon} d_{\nu}$ provides an asymptotically uniformly most powerful test of limiting size $\epsilon$ and of asymptotic power (1.20). Discussion of the $M_{\nu}$-test will be continued in Section 6 .

3.5. Transformation of the observations. This may considerably enlarge the scope of the regression model (1.1). For example, if

$$
P\left(X_{\nu i} \leqq x\right)=F\left[x^{1 / \sigma} \exp \left(\left(-\alpha-\beta c_{\nu i}\right) / \sigma\right)\right],
$$

where $F(0)=0$, then we have

$$
P\left(\log X_{\nu i} \leqq y\right)=F\left[\exp \left(\left(y-\alpha-\beta c_{\nu i}\right) / \sigma\right)\right],
$$

so that the transformed observations $\log X_{\nu i}$ obey the model (1.1) with $F(y)$ replaced by $F(\exp (y))$. Just in this way we have obtained the density No. 4 in Table 1 from the density $e^{-y}, y>0$.

If (3.8) still holds, and the distribution is symmetrical about zero (i.e., $F(x)+F(-x)=1)$, then the absolute values $\left|X_{\nu 1}\right|, \cdots,\left|X_{\nu_{\nu}}\right|$ represent a sufficient statistic. Furthermore, we have

$$
P\left(\log \left|X_{v i}\right| \leqq y\right)=2 F\left[\exp \left(\left(y-\alpha-\beta c_{v i}\right) / \sigma\right)\right]-1,
$$

so that the transformed observations $\log \left|X_{\nu i}\right|$ obey the model (1.1) with $F(y)$ replaced by $2 F(\exp (y))-1$.

In this manner our model may be used not only to detect location trends but also to detect scalar trends in one-sided and symmetrical distributions. So, for example, all particular cases considered by Capon [1] fall within the scope of model (1.1). Paper [1] is methodically based on the paper by Chernoff and Savage [2]. From the point of view of our particular problem, however, the assumptions imposed in [2] are too restrictive.

3.6. If $\sigma$ were known, say $\sigma=1$, all assertions would remain valid. If $\alpha$ were known, then we would have to suppose that $N_{\nu} \bar{c}_{\nu}^{2}\left(\sum_{i=1}^{N_{\nu}} c_{\nu i}^{2}\right)^{-1} \rightarrow 0$.

3.7. In order to remove assumption (1.5), we need to prove that $d_{\nu} \rightarrow \infty$ entails the power of test (1.16) tends to 1 . It is very plausible, but we failed to prove it.

3.8. Given the function $\varphi(u)$, we can construct $f(x)$ as follows. First we establish

$$
f(u)=-\int_{-\infty}^{u} \varphi(\lambda) d \lambda,
$$

and then, provided the integral exists,

$$
x(u)=\int_{\frac{1}{2}}^{u} d \lambda / f(\lambda) .
$$

The integral (3.11) exists, for example, if $f(u)$ is positive for $0<u<1$. Then, upon eliminating the parameter $u$, we get $f(x)$.

3.9. The distributions with non-decreasing $\varphi(u)$ are called strongly unimodal. 
Ibragimov [7] proved that the convolution of $f(x)$ with every unimodal $g(x)$ is a unimodal distribution, if and only if $f(x)$ is strongly unimodal.

\section{Preliminaries.}

Contiguity. Let $\left\{P_{v}\right\}$ and $\left\{Q_{v}\right\}$ be two sequences of probability measures defined on a sequence of measurable spaces $\left\{\mathfrak{X}_{\nu}, \mathfrak{A}_{\nu}\right\}, 1 \leqq \nu<\infty$.

Definition 4.1. If for any sequence of events $\left\{A_{\nu}\right\}, A_{\nu} \varepsilon \mathfrak{A}_{\nu}, P_{\nu}\left(A_{\nu}\right) \rightarrow 0$ entails $Q_{\nu}\left(A_{\nu}\right) \rightarrow 0$, we say that the probability measures $Q_{\nu}$ are contiguous to the probability measures $P_{\nu}, 1 \leqq \nu<\infty$.

Contiguity implies that the singular part of $Q_{\nu}$ tends to zero as $\nu \rightarrow \infty$, and that any sequence of random variables $\left\{Y_{\nu}\right\}$ converging to $Y$ in $P_{\nu}$-probability converges to $Y$ in $Q_{\nu}$-probability as well.

Let $\left\{B_{\nu}\right\}$ be a sequence of events such that $P_{\nu}\left(B_{\nu}\right)=1$ and $Q_{\nu}$ is absolutely continuous with respect to $P_{\nu}$ on $B_{\nu}, 1 \leqq \nu<\infty$. Put

$$
\begin{aligned}
& r_{\nu}=d Q_{\nu} / d P_{\nu} \text { on } B_{\nu}, \\
& =0 \quad \text { on } \mathfrak{X}_{\nu}-B_{\nu} \text {, }
\end{aligned}
$$

where $d Q_{\nu} / d P_{\nu}$ denotes the usual Radon-Nikodym derivative. The probability measures $Q_{\nu}$ are contiguous to the probability measures $P_{\nu}$, if and only if, first,

$$
\lim _{\nu \rightarrow \infty} \int r_{\nu} d P_{\nu}=\lim _{\nu \rightarrow \infty} Q_{\nu}\left(B_{\nu}\right)=1,
$$

and, second, to any $\epsilon>0$ there exists $a \delta>0$ such that

$$
\left[P_{\nu}\left(A_{\nu}\right)<\delta\right]=>\left[\int_{A_{\nu}} r_{\nu} d P_{\nu}=Q_{\nu}\left(A_{\nu} \cap B_{\nu}\right)<\epsilon\right], \quad 1 \leqq \nu<\infty .
$$

Condition (4.3) means, however, that the likelihood ratios $r_{\nu}$ are uniformly integrable.

Let us mention two well-known criteria for uniform integrability of a sequence of functions $\left\{h_{v}\right\}$. First, one called the Vallee Poussin theorem, is based on boundedness of integrals

$$
\sup _{\nu} \int\left|h_{\nu}\right| \Psi\left(\left|h_{\nu}\right|\right) d P_{\nu}<\infty,
$$

where $\Psi(t), t \geqq 0$, is any function such that $\Psi(t) \uparrow \infty$, if $t \rightarrow \infty$, and $t \Psi(t)$ is bounded from below. For example, we can take $\Psi(t)=t$, or $\log t$.

The second criterion may be formulated as follows: If $P_{\nu}=P, h_{\nu}$ are nonnegative and $h_{\nu} \rightarrow h$ in $P$-probability, then the sequence $\left\{h_{v}\right\}$ is uniformly $P$ integrable, if, and only if,

$$
\lim _{\nu \rightarrow \infty} \int h_{\nu} d P=\int h d P
$$

Observe that from the Fatou lemma it follows

$$
\lim _{\nu \rightarrow \infty} \inf \int h_{\nu} d P \geqq \int h d P .
$$


LeCam [8] recognized that the condition $P_{\nu}=P$ may be avoided, if we replace the convergence in probability by convergence in distribution. He derived a basic result, which will be presented below as Lemma 4.1.

Denote

$$
F_{\nu}(x)=P_{\nu}\left(r_{\nu} \leqq x\right) .
$$

The sequence $\left\{r_{\nu}\right\}$ converges in distribution, if there exists a distribution function $F(x)$ such that

$$
\lim _{\nu \rightarrow \infty} F_{\nu}(x)=F(x)
$$

in all continuity of points of $F(x)$. Since

$$
1 \geqq Q_{\nu}\left(r_{\nu}>x\right) \geqq x P_{\nu}\left(r_{\nu}>x\right)=x\left[1-F_{\nu}(x)\right],
$$

it holds that

$$
F_{\nu}(x) \geqq 1-(1 / x), \quad x>0 .
$$

From (4.8) it follows that the sequence $\left\{F_{\nu}\right\}$ is relatively compact, i.e., from every subsequence $\{k\} \subset\{\nu\}$ we can draw a further subsequence $\{j\} \subset\{k\}$ such that (4.7) holds true for $\nu$ running through $\{j\}$, and every $F(x)$ such that (4.7) holds true is a distribution function.

Lemma 4.1 (Le Cam). Keep the above notations. The probability measures $Q_{\nu}$ are contiguous to the probability measures $P_{\nu}$, if, and only if, for any subsequence $\{j\} \subset\{\nu\}, F_{j}(x) \rightarrow F(x)$ in continuity points of $F(x)$ implies

$$
\int_{-\infty}^{\infty} x d F(x)=1
$$

Proof.

Sufficiency. Suppose that, to the contrary, for some subsequence $\{k\} \subset\{\nu\}$ there exist a sequence of events $\left\{A_{k}\right\}$ and a positive number $\epsilon>0$ such that $P_{k}\left(A_{k}\right) \rightarrow 0$ and

$$
Q_{k}\left(A_{k}\right) \geqq \epsilon, \quad k \varepsilon\{k\} .
$$

Obviously, we can assume that the events are of the form $A_{k}=\left\{r_{k} \geqq x_{k}\right\}$ with $x_{k} \rightarrow \infty$. Draw a further subsequence $\{j\} \subset\{k\}$ such that $F_{j}(x) \rightarrow F(x)$ in continuity points of $F(x)$. If $x_{0}$ is a continuity point of $F(x)$, then from (4.10) and $A_{j}=\left\{r_{j} \geqq x_{j}\right\}$ it follows that

$$
\begin{aligned}
1-\epsilon & \geqq \lim _{\sup _{j \rightarrow \infty}} Q_{j}\left(r_{j}<x_{j}\right) \\
& \geqq \lim _{j \rightarrow \infty} Q_{j}\left(r_{j}<x_{0}\right)=\lim _{j \rightarrow \infty} \int_{\left\{r_{j}<x_{0}\right\}} r_{j} d P_{j} \\
& =\lim _{j \rightarrow \infty} \int_{-0}^{x_{0}} x d F_{j}(x)=\int_{-0}^{x_{0}} x d F(x),
\end{aligned}
$$

which contradicts (4.9), since $x_{0}$ may be arbitrarily large. 
Necessity. Suppose that for some $\epsilon>0$

$$
\int_{-0}^{\infty} x d F(x)<1-2 \epsilon,
$$

where $F^{\prime}(x)$ is the limit of subsequence $\left\{F_{j}\right\}$. Now, if

$$
Q_{j}\left(B_{j}\right)=\int_{-0}^{\infty} x d F_{j}(x) \nrightarrow 1,
$$

the proof is finished. In the opposite case, for every continuity point $x_{0}$

$$
Q_{j}\left(r_{j}>x_{0}\right)=\int_{x_{0}}^{\infty} x d F_{j}(x) \rightarrow 1-\int_{-0}^{x_{0}} x d F(x) \geqq 2 \epsilon
$$

which entails the existence of random events $A_{j}=\left\{r_{j}>x_{j}\right\}, x_{j} \rightarrow \infty$, such that (4.10) holds true, whereas $P\left(r_{j}>x_{j}\right)=1-F_{j}(x) \rightarrow 0$, on account of $x_{j} \rightarrow \infty$ and (4.8). The proof is terminated.

Now we shall analyse the most important case where $P_{\nu}$ and $Q_{\nu}$ are two probability distributions of a vector $\left(X_{\nu 1}, \cdots, X_{\nu N_{\nu}}\right)$, whose components are independent. In this case, under rather general conditions, the only possible limits of subsequences $\left\{F_{j}\right\}$ are log-normal distributions. Let us have

$$
\begin{aligned}
& P_{\nu}\left(X_{\nu i} \leqq x_{i}, 1 \leqq i \leqq N_{\nu}\right)=\prod_{i=1}^{N_{\nu}} P_{\nu i}\left(X_{\nu i} \leqq x_{i}\right) \\
& Q_{\nu}\left(X_{\nu i} \leqq x_{i}, 1 \leqq i \leqq N_{\nu}\right)=\prod_{i=1}^{N_{\nu}} Q_{\nu i}\left(X_{\nu i} \leqq x_{i}\right)
\end{aligned}
$$

and put

$$
r_{\nu i}=d Q_{\nu i} / d P_{\nu i}, \quad 1 \leqq i \leqq N_{\nu}, 1 \leqq \nu<\infty,
$$

on sets $B_{v i}$ such that $P_{\nu i}\left(B_{v i}\right)=1$ and $Q_{v i}$ is absolutely continuous with respect to $P_{\nu i}$, otherwise put $r_{v i}=0$. If $p_{v i}(x)$ and $q_{v i}(x)$ are densities corresponding to distribution functions $P_{\nu i}$ and $Q_{\nu i}$, respectively, then

$$
r_{v i}(x)=q_{v i}(x) / p_{\nu i}(x), \quad \quad \text { if } p_{v i}(x)>0,
$$

and $r_{\nu i}(x)=0$ otherwise.

Introduce the statistics.

$$
W_{\nu}=2 \sum_{i=1}^{N_{\nu}}\left(\left(r_{\nu i}\right)^{\frac{1}{2}}-1\right)
$$

and

$$
L_{v}=\sum_{i=1}^{N_{\nu}} \log r_{v i},
$$

and suppose that for every $\epsilon>0$

$$
\lim _{\nu \rightarrow \infty} \max _{1 \leqq i \leqq N_{\nu}} P_{\nu i}\left(\left|r_{\nu i}-1\right|>\epsilon\right)=0 .
$$


Let $\mathfrak{R}\left[Y_{\nu} \mid P_{\nu}\right] \rightarrow \mathfrak{N}\left[a_{\nu}, b_{\nu}^{2}\right]$ denote that the distribution of $b_{\nu}^{-1}\left(Y_{\nu}-a_{\nu}\right)$ under $P_{\nu}$ tends to the normal distribution with mean 0 and variance 1 . A similar meaning will be ascribed to $\mathfrak{R}\left[Y_{\nu} \mid Q_{\nu}\right] \rightarrow \mathfrak{N}\left[a_{\nu}, b_{\nu}^{2}\right]$.

The following lemma has been compiled from LeCam's results [8], [9].

Lemma 4.2 (Le Cam). Assume that (4.17) holds true and that $\mathbb{2}\left[W_{\nu} \mid P_{\nu}\right] \rightarrow$ $\mathfrak{N}\left[-\frac{1}{4} \sigma^{2}, \sigma^{2}\right]$. Then

(1) the probability distributions $Q_{\nu}$ are contiguous to the probability measures $P_{\nu}$

(2) $W_{\nu}-L_{\nu} \rightarrow \frac{1}{4} \sigma^{2}$ in $P_{\nu}$-probability and $\Re\left[L_{\nu} \mid P_{\nu}\right] \rightarrow \mathfrak{N}\left[-\frac{1}{2} \sigma^{2}, \sigma^{2}\right]$,

(3) if $\&\left[Y_{\nu} \mid P_{\nu}\right] \rightarrow \mathfrak{N}\left[a, b^{2}\right]$ and $\mathfrak{Q}\left[Y_{\nu}, L_{\nu} \mid P_{\nu}\right]$ tends to a bivariate normal distribution with correlation coefficient $\rho$, then

$$
\mathfrak{R}\left[Y_{\nu} \mid Q_{\nu}\right] \rightarrow \mathfrak{N}\left[a+\rho b \sigma, b^{2}\right] .
$$

Proof. Upon setting $u_{\nu_{i}}=2\left(\left(r_{\nu i}\right)^{\frac{1}{2}}-1\right)$, we easily find that

$$
L_{\nu}=W_{\nu}-\frac{1}{4} \sum_{i=1}^{N_{\nu}} u_{\nu i}^{2} \int_{0}^{1}\left[2(1-\lambda) d \lambda /\left(1+\frac{1}{2} \lambda u_{\nu i}\right)^{2}\right]
$$

Now, on account of (4.17) and of convergence of $W_{\nu}$ to the normal distribution, we have for any $\epsilon>0$

$$
\lim _{\nu \rightarrow \infty} P\left\{\max _{1 \leqq i \leqq N_{\nu}}\left|u_{\nu i}\right|>\epsilon\right\}=0,
$$

which implies that the last term in (4.19) has the same limiting distribution as $\frac{1}{4} \sum_{i=1}^{N_{\nu}} u_{\nu i}^{2}$, if any. Furthermore, the limiting distribution of $\sum_{i=1}^{N_{\nu}} u_{\nu i}^{2}$ will be the same as that of $\sum_{i=1}^{N_{\nu}} u_{\nu i}^{2}(\delta)$, where

$$
\begin{aligned}
u_{\nu i}(\delta) & =u_{\nu i}, \quad \text { if }\left|u_{\nu i}\right|<\delta, \\
& =0 \text { otherwise. }
\end{aligned}
$$

For any $\delta>0$ the mean value of $\sum_{i=1}^{N_{\nu}} u_{\nu i}^{2}(\delta)$ equals asymptotically $\sigma^{2}$ and the variance is clearly less than $\delta^{2} \sigma^{2}$. This implies that $\sum_{i=1}^{N_{\nu}} u_{\nu i}^{2} \rightarrow \sigma^{2}$ in $P_{\nu}$-probability, which concluded the proof of assertion (2).

Since $r_{\nu}=\exp \left(L_{\nu}\right),(2)$ entails $F_{\nu}(x) \rightarrow F(x)$, where $F\left(e^{y}\right)$ is the normal distribution function with parameters $\left[-\frac{1}{2} \sigma^{2}, \sigma^{2}\right]$. This implies that (4.9) holds true, and hence, according to Lemma 4.1, assertion (1) holds true.

It remains to prove $(3)$. Let $F_{\nu}(u, v)$ be the distribution function of $\left(Y_{\nu}, L_{\nu}\right)$ and $F(u, v)$ be the limiting bivariate normal distribution. Obviously,

$$
\begin{aligned}
Q_{\nu}\left(Y_{\nu}<x\right) & =\int_{\left\{Y_{\nu}<x\right\}} r_{\nu} d P_{\nu}+Q_{\nu}\left(A_{\nu}\right) \\
& =\int_{-\infty}^{x} \int_{-\infty}^{\infty} e^{v} d F_{\nu}(u, v)+Q_{\nu}\left(A_{\nu}\right),
\end{aligned}
$$

where $Q_{\nu}\left(A_{\nu}\right) \rightarrow 0$ because of contiguity. For the same reason, the $r_{\nu}$ 's are uniformly integrable, and hence

$$
\lim _{\nu \rightarrow \infty} \int_{-\infty}^{x} \int_{-\infty}^{\infty} e^{v} d F_{\nu}(u, v)=\int_{-\infty}^{x} \int_{-\infty}^{\infty} e^{v} d F(u, v)
$$


Now, after easy computations,

$$
\begin{aligned}
& \int_{-\infty}^{x} \int_{-\infty}^{\infty} e^{v} d F(u, v)=\int_{-\infty}^{x} \int_{-\infty}^{\infty} b^{-1} \sigma^{-1}\left[2 \pi\left(1-\rho^{2}\right)\right]^{-\frac{1}{2}} \\
& \cdot \exp \left\{v-\left[2\left(1-\rho^{2}\right)\right]^{-1}\left\{\left[(u-a)^{2} / b^{2}\right]-\left[2 \rho(u-a)\left(v+\frac{1}{2} \sigma^{2}\right) / b \sigma\right]+\left[\left(v+\frac{1}{2} \sigma^{2}\right)^{2} / \sigma^{2}\right]\right\}\right\} \\
& \quad=b^{-1}(2 \pi)^{-\frac{1}{2}} \int_{-\infty}^{x} \exp \left[-\frac{1}{2}\left((u-a-\rho b \sigma)^{2} / b^{2}\right)\right] d u,
\end{aligned}
$$

which concludes the proof.

REMARK 4.1. The notion of contiguity has been developed independently by LeCam and the present author. In the original version of this paper the less efficient criterion (4.4) had been used, which lead to much weaker results. Then, under the influence of LeCam's ideas, the paper was revised and put in the present form.

In conclusion, we formulate a lemma concerning functions possessing a quadratically integrable derivative.

Lemma 4.3. Let $s(x),-\infty<x<\infty$, be an absolutely continuous function possessing a quadratically integrable derivative $s^{\prime}(x)$. Then

$$
\lim _{h \rightarrow 0} \int_{-\infty}^{\infty}\left\{([s(x-h)-s(x)] / h)-s^{\prime}(x)\right\}^{2} d x=0
$$

Proof. From

$$
\{[s(x-h)-s(x)] / h\}^{2}=\left[(1 / h) \int_{x-h}^{x} s^{\prime}(y) d y\right]^{2} \leqq(1 / h) \int_{x-h}^{x}\left[s^{\prime}(y)\right]^{2} d y
$$

it follows that

$$
\int_{-\infty}^{\infty}\{[s(x-h)-s(x)] / h\}^{2} d x \leqq \int_{-\infty}^{\infty}\left[s^{\prime}(x)\right]^{2} d x .
$$

Furthermore, $h^{-1}[s(x)-s(x-h)] \rightarrow s^{\prime}(x)$ almost surely, so that, due to (4.23) and Fatou's lemma,

$$
\lim _{h \rightarrow 0} \int_{-\infty}^{\infty}\{[s(x-h)-s(x)] / h\}^{2} d x=\int_{-\infty}^{\infty}\left[s^{\prime}(x)\right]^{2} d x
$$

However, (4.24) implies, according to the above-mentioned criterion (4.5), that the functions $h^{-2}[s(x)-s(x-h)]^{2}$ are uniformly integrable, which entails (4.22). The proof is finished.

5. Proof of the main theorem. Put

$$
s(x)=(f(x))^{\frac{1}{2}},
$$

so that

$$
s^{\prime}(x)=\frac{1}{2}\left[f^{\prime}(x) /(f(x))^{\frac{1}{2}}\right] .
$$

Consequently, if (1.3) is satisfied, $s(x)$ has a quadratically integrable derivative. 
Now take a particular alternative, say $\left[\alpha=\alpha_{0}, \beta=\beta_{0}, \sigma=\sigma_{0}\right]$, and associate with it the particular hypothesis $\left[\alpha=\alpha_{0}+\beta_{0} \bar{c}_{\nu}, \beta=0, \sigma=\sigma_{0}\right]$. Denoting the distribution under the alternative and the hypothesis by $Q_{\nu}$ and $P_{\nu}$, respectively, we can express the statistic (4.15) in the form

$$
W_{\nu}=2 \sum_{i=1}^{N_{\nu}}\left\{\left(\left[s\left(Y_{\nu i}-\gamma c_{\nu i}+\gamma \bar{c}_{\nu}\right)\right] / s\left(Y_{\nu i}\right)\right)-1\right\}
$$

where

$$
Y_{\nu i}=\left[X_{\nu i}-\alpha_{0}-\beta_{0} \bar{c}_{\nu}\right] / \sigma_{0}
$$

and

$$
\gamma=\beta_{0} / \sigma_{0}
$$

Let $E$ denote the mean value referring to $P_{\nu}$. We easily see that, because of Lemma 4.3,

$$
\begin{aligned}
2 E\left[\frac{s\left(Y_{\nu i}-h\right)}{s\left(Y_{\nu i}\right)}-1\right] & =-h^{2} \int_{-\infty}^{\infty}\left[\frac{s(x-h)-s(x)}{h}\right]^{2} d x \\
& \sim-h^{2} \int_{-\infty}^{\infty}\left[s^{\prime}(x)\right]^{2} d x=-h^{2} \int_{-\infty}^{\infty}\left[f^{\prime}(x) / f(x)\right]^{2} f(x) d x
\end{aligned}
$$

where the sign $\sim$ denotes that the ratio of both sides tends to 1 as $h \rightarrow 0$. Consequently, in view of (5.3),

$$
E W_{\nu} \sim-\frac{1}{4} \gamma^{2} d_{\nu}^{2}
$$

where $\gamma$ and $d_{\nu}^{2}$ are given by (5.5) and (1.12).

Now we shall approximate $W_{\nu}$ by

$$
T_{\nu}=\sum_{i=1}^{N_{\nu}}\left(c_{\nu i}-\bar{c}_{\nu}\right)\left[f^{\prime}\left(Y_{\nu i}\right) / f\left(Y_{\nu i}\right)\right]
$$

Under the hypothesis, obviously,

$$
\operatorname{Var} T_{\nu}=d_{\nu}^{2} .
$$

Furthermore, upon setting $h_{\nu i}=\gamma c_{\nu i}-\gamma \bar{c}_{\nu}$,

$$
\begin{array}{r}
E\left(W_{\nu}-E W_{\nu}-\gamma T_{\nu}\right)^{2} \leqq 4 \sum_{i=1}^{N_{\nu}} E\left[\frac{s\left(Y_{\nu i}-h_{\nu i}\right)}{s\left(Y_{\nu i}\right)}-1-\frac{1}{2} h_{\nu i} \frac{f^{\prime}\left(Y_{\nu i}\right)}{f\left(Y_{\nu i}\right)}\right]^{2} \\
\leqq 4 \gamma^{2} \sum_{i=1}^{N_{\nu}}\left(c_{\nu i}-\bar{c}_{\nu}\right)^{2} \int\left[\frac{s\left(x-h_{\nu i}\right)-s(x)}{h_{\nu i}}-s^{\prime}(x)\right]^{2} d x
\end{array}
$$

From (1.5), (5.9), (5.10) and Lemma 4.3 it follows that

$$
\lim _{\nu \rightarrow \infty} E\left(W_{\nu}-E W_{\nu}-\gamma T_{\nu}\right)^{2}=0 .
$$

Furthermore, introducing the statistic

$$
T_{\nu}^{*}=\sum_{i=1}^{N_{\nu}}\left(c_{\nu i}-\bar{c}_{\nu}\right) \varphi_{\nu}\left(F\left(Y_{\nu i}\right)\right),
$$


and consulting (1.19), we can see that

(5.13) $\lim _{\nu \rightarrow \infty} E\left(T_{\nu}-T_{\nu}^{*}\right)^{2} \leqq \lim _{\nu \rightarrow \infty} \sum_{\nu=1}^{N_{\nu}}\left(c_{\nu i}-\bar{c}_{\nu}\right)^{2} \int_{0}^{1}\left[\varphi_{\nu}(u)-\varphi(u)\right]^{2} d u=0$.

Now, (5.7), (5.11) and (5.13) imply

$$
P_{\nu} \lim _{\nu \rightarrow \infty}\left(W_{\nu}-\frac{1}{4} \gamma^{2} d_{\nu}^{2}-\gamma T_{\nu}\right)=0
$$

and

$$
P_{\nu} \lim _{\nu \rightarrow \infty}\left(T_{\nu}^{*}-T_{\nu}\right)=0,
$$

where $P_{\nu} \lim$ denotes the limit in $P_{\nu}$-probability. Furthermore, according to Theorem 3.1 of [6],

$$
\lim _{\nu \rightarrow \infty} E\left(S_{\nu}-T_{\nu}^{*}\right)^{2}=0,
$$

which in conjunction with (5.13) implies

$$
P_{\nu} \lim _{\nu \rightarrow \infty}\left(S_{\nu}-T_{\nu}\right)=0 .
$$

However, it is easy to see ([5], Th. 4, p. 103), that

$$
\mathfrak{R}\left[T_{\nu} \mid P_{\nu}\right] \rightarrow \mathfrak{N}\left[0, d_{\nu}^{2}\right],
$$

and, consequently, on account of (5.14),

$$
\mathfrak{R}\left(W_{\nu} \mid P_{\nu}\right) \rightarrow \mathfrak{N}\left[-\frac{1}{4} \gamma^{2} d_{\nu}^{2}, \gamma^{2} d_{\nu}^{2}\right]
$$

Now observe that if the assertion of Theorem 1.1 were not true, there would exist a subsequence $\{j\} \subset\{\nu\}$ such that $d_{j}^{2} \rightarrow d^{2}$, and that the assertion would still be false for the problem reduced to this subsequence $\{j\}$. So we may assume, without any loss of generality, that

$$
\lim _{\nu \rightarrow \infty} d_{\nu}^{2}=d^{2} \text {. }
$$

Upon making use of Lemma 4.1, we conclude that the distributions $Q_{v}$ are contiguous to the distributions $P_{\nu}$, and that

$$
P_{\nu} \lim _{\nu \rightarrow \infty}\left(L_{\nu}-\frac{1}{2} \gamma^{2} d^{2}-\gamma T_{\nu}\right)=0
$$

Now (5.21), (5.17) and contiguity imply that

$$
Q_{\nu} \lim _{\nu \rightarrow \infty}\left(L_{\nu}-\frac{1}{2} \gamma^{2} d^{2}-\gamma S_{\nu}\right)=0
$$

Consequently, the best critical region for distinguishing the particular alternative $Q_{\nu}$ from the particular hypothesis $P_{\nu}$, known to be based on the statistic $L_{\nu}$ (Neyman-Pearson lemma), may be asymptotically approximated by the test (1.16). Since the statistic $S_{\nu}$ is distribution free, the test has the correct limiting size for all possible particular hypotheses, and, consequently, it is really asymptotically uniformly most powerful.

It remains to find the limit of $\mathfrak{R}\left[S_{\nu} \mid Q_{\nu}\right]$. From contiguity and (5.17) it follows that, if one of the limits exists,

$$
\lim _{\nu \rightarrow \infty} \mathfrak{R}\left[S_{\nu} \mid Q_{\nu}\right]=\lim _{\nu \rightarrow \infty} \mathfrak{R}\left[T_{\nu} \mid Q_{\nu}\right] .
$$


However, as we have seen, $\&\left[T_{\nu} \mid P_{\nu}\right] \rightarrow \mathfrak{N}\left[0, d^{2}\right]$, and, further, on account of (5.21) $\&\left[L_{\nu}, T_{\nu} \mid P_{\nu}\right]$ tends to a degenerated bivariate normal distribution with correlation coefficient $\rho=1$ and variances $\gamma^{2} d^{2}$ and $d^{2}$ referring to $L_{\nu}$ and $T_{\nu}$, respectively. Consequently, according to assertion (3) of Lemma 4.1,

$$
\lim _{\nu \rightarrow \infty} \mathfrak{R}\left[T_{\nu} \mid Q_{\nu}\right]=\mathfrak{N}\left[\gamma d^{2}, d^{2}\right] .
$$

Now, from (5.23) and (5.24) we easily conclude (1.20), and the proof is completed.

6. Asymptotic efficiency. If the true density, say $g(x)$, differs from the supposed one, say $f(x)$, then the tests based on $S_{\nu}$ will be no longer asymptotically most powerful. Denote

$$
\psi(u)=-\left[g^{\prime}\left(G^{-1}(u)\right) / g\left(G^{-1}(u)\right)\right],
$$

where $G(x)=\int_{-\infty}^{x} g(y) d y$, so that $\psi(u)$ parallels $\varphi(u)$, given by (1.6). Assume that

$$
\int_{0}^{1} \psi^{2}(u) d u=\int_{-\infty}^{\infty}\left[g^{\prime}(x) / g(x)\right]^{2} g(x) d x<\infty,
$$

and introduce the correlation coefficient

$$
\rho=\left[\int_{0}^{1} \varphi(u) \psi(u) d u\right] /\left[\int_{0}^{1} \varphi^{2}(u) d u \int_{0}^{1} \psi^{2}(u) d u\right]^{\frac{1}{2}} .
$$

Theorem 6.1. Let the conditions (1.3), (1.4), (1.5), (1.19) and (6.2) be satisfied. Then the asymptotic power of the test $S_{\nu}>K_{\mathrm{\epsilon}} d_{\nu}$ equals

$$
1-\Phi\left(K_{\epsilon}-\rho(\beta / \sigma) d_{\nu}\right),
$$

where $\rho$ is given by (6.3).

Proof. Let $P_{\nu}$ correspond to $G$ and to $\alpha=\alpha_{0}+\beta_{0} \bar{c}_{\nu}, \beta=0, \sigma=\sigma_{0}$, and let $Q_{\nu}$ correspond to $G$ and $\alpha=\alpha_{0}, \beta=\beta_{0}, \sigma=\sigma_{0}$. Introduce statistics

$$
L_{\nu}=\sum_{i=1}^{N_{\nu}} \log \left[g\left(Y_{\nu i}-\gamma c_{\nu i}+\gamma \bar{c}_{\nu}\right) / g\left(Y_{\nu i}\right)\right],
$$

and

$$
T_{\nu}=\sum_{i=1}^{N_{\nu}}\left(c_{\nu i}-\bar{c}_{\nu}\right) \varphi\left(G\left(Y_{\nu i}\right)\right) .
$$

Now, upon following literally the pattern used in proving Theorem 1.1, we prove that (5.23) still holds with out redefined $Q_{\nu}$ and $T_{\nu}$, and that $\Re\left[L_{\nu}, T_{\nu} \mid P_{\nu}\right]$ tends to the bivariate normal distribution with correlation coefficient $\rho$ given by (6.3). Hence the result.

The number $\rho^{2}$ is usually called the efficiency. In the two-sample problem where $n_{\nu} / N_{\nu} \rightarrow \lambda, 0<\lambda<1$, the efficiency may be interpreted as the ratio of sample sizes needed to attain the same power. In the general regression problem the sample size $N_{\nu}$ should be replaced by the sum $\sum_{i=1}^{N_{\nu}}\left(c_{\nu i}-\bar{c}_{\nu}\right)^{2}$. However, even 
then the interpretation fails if $\rho<0$. This case may arise only if $\varphi$ and $\psi$ both are non-monotoneous, for example, if

$$
\begin{aligned}
\varphi(u) & =-u, \\
& =u-\frac{1}{2}, \\
& =1-u,
\end{aligned}
$$

$$
\begin{aligned}
& 0<u \leqq \frac{1}{4}, \\
& \frac{1}{4}<u \leqq \frac{3}{4}, \\
& \frac{3}{4}<u<1
\end{aligned}
$$

and

$$
\begin{aligned}
\psi(u) & =-u, \\
& =\frac{1}{64}, \\
& =-\frac{1}{64}, \\
& =u
\end{aligned}
$$$$
0<u \leqq \frac{1}{8} \text {, }
$$$$
\frac{1}{8}<u \leqq \frac{1}{2} \text {, }
$$$$
\frac{1}{2}<u \leqq \frac{7}{8} \text {, }
$$$$
\frac{7}{8}<u<1 \text {. }
$$

The functions $f(u)=-\int_{0}^{u} \varphi(\lambda) d \lambda$ and $g(u)=-\int_{0}^{u} \psi(\lambda) d \lambda$ are positive for $0<u<1$, so that, according to Subsection 3.8, the corresponding densities exist. However, the correlation coefficient equals

$$
\rho=-(10 / 16 \sqrt{73}),
$$

so that the asymptotic power is less than the limiting size, provided that $\lim _{\nu \rightarrow \infty}$ inf $d_{\nu}>0$. So, in a wider model, where $F$ is not known, the $S_{\nu}(\varphi)$-test with non-monotoneous $\varphi$ may not be unbiased.

Now we shall compare the efficiency of the rank-order tests with the efficiency of the test based on Neyman's statistic $M_{\nu}$ given by (3.4). Suppose that there exist numbers $\alpha(g)$ and $\sigma(g)$ such that

$$
\lim _{v \rightarrow \infty} E\left[\frac{\frac{f^{\prime}\left(X_{\nu 1}-\hat{\alpha}\right)}{\hat{\sigma}}}{f\left(\frac{X_{\nu 1}-\hat{\alpha}}{\hat{\sigma}}\right)}-\frac{f^{\prime}\left(\frac{X_{\nu 1}-\alpha(g)}{\sigma(g)}\right)}{f\left(\frac{X_{\nu 1}-\alpha(g)}{\sigma(g)}\right)}\right]^{2}=0
$$

where $E$ refers to the hypothesis that $P\left(X_{\nu i}<x\right)=G(x), 1 \leqq i \leqq N_{\nu}$, $1 \leqq \nu<\infty$. Moreover, in concordance with (3.5) and (3.6) let us assume that

$$
\alpha\left(g_{a, b}\right)=b \alpha(g)+a,
$$

and

$$
\sigma\left(g_{a, b}\right)=b \sigma(g)
$$

where

$$
g_{a, b}(x)=(1 / b) g((x-a) / b), \quad-\infty<a<\infty, \quad b>0 .
$$

Put $g_{0}=g_{a, b}$ with $a$ and $b$ chosen so that

$$
\alpha\left(g_{0}\right)=0, \quad \sigma\left(g_{0}\right)=1 .
$$

Introduce another correlation coefficient

$$
\rho^{*}=C /(A B)^{\frac{1}{2}},
$$


where

$$
\begin{gathered}
A=\int_{-\infty}^{\infty}\left[g_{0}^{\prime}(x) / g_{0}(x)\right]^{2} g_{0}(x) d x \\
B=\int_{-\infty}^{\infty}\left[f^{\prime}(x) / f(x)\right]^{2} g_{0}(x) d x-\left\{\int_{-\infty}^{\infty}\left[f^{\prime}(x) / f(x)\right] g_{0}(x) d x\right\}^{2}
\end{gathered}
$$

and

$$
C=\int_{-\infty}^{\infty}\left[f^{\prime}(x) / f(x)\right]\left[g_{0}^{\prime}(x) / g_{0}(x)\right] g_{0}(x) d x
$$

Under the above assumption, the asymptotic power of Neyman's $M_{\nu}$-test equals

$$
1-\Phi\left(K_{\epsilon}-\rho^{*}(\beta / \sigma) d_{\nu}\right) \text {. }
$$

An interesting question arises: what is the relation of $\rho$ and $\rho^{*}$ ? If $f$ is the normal density, then Chernoff and Savage [2] proved that

$$
\rho^{*} \leqq \rho,
$$

with equality only if $g \equiv f$ up to a change of location and scale. Directly from the definition of $\rho^{*}$ it follows that it does not depend on changes of location and scale (the same is true of $\rho$, because of (1.9)). If $f$ is normal and $\hat{\alpha}=\bar{x}$ and $\hat{\sigma}=s$, where $s$ is the sample standard deviation, then (6.11) means that in computing $\rho^{*}$ according to (6.12) through (6.15) we restrict outselves to densities with zero means and unity variances. The thesis by Mikulski ([10], remarks on p. 17) strongly supports the conjecture that (6.17) hold true generally. To avoid misunderstandings, let us point out that Mikulski's paper aims at disproving the conjecture and succeeds in doing it in the two-sample problem, where, however, nuisance parameters are not involved. Upon introducing the nuisance parameters $\alpha$ and $\sigma$ and using invariant estimates $\hat{\alpha}$ and $\hat{\sigma}$, the distribution of the statistic $M_{\nu}$ becomes independent of changes of location and scale, which brings it closer to the statistic $S_{\nu}$. The solution of the corresponding variational problem is then to be chosen in the class of densities satisfying the side conditions (6.11). The author hopes to explore the question in a subsequent paper.

7. Tests of symmetry. Let $f(x)$ be symmetric density, i.e.,

$$
f(x)=f(-x), \quad-\infty<x<\infty .
$$

Consider the hypothesis that the $X_{\nu i}$ 's are independent and have a common probability density $f(x / \sigma)$, where $\sigma$ is a nuisance parameter $(\sigma>0)$, against the alternative that the densities equal $f\left(\left(x-\beta c_{\nu i}\right) / \sigma\right)$, where

$$
\lim _{\nu \rightarrow \infty}\left\{\max _{1 \leqq i \leqq N_{\nu}} c_{\nu i}^{2} / \sum_{i=1}^{N_{\nu}} c_{\nu i}^{2}\right\}=0
$$


and

$$
\sup _{\nu} \sum_{i=1}^{N_{\nu}} c_{\nu i}^{2}<\infty
$$

Now let $R_{\nu i}$ be the rank of $\left|X_{\nu i}\right|$ in the sequence $\left|X_{\nu i}\right|, \cdots,\left|X_{\nu N_{\nu}}\right|$ rearranged according to ascending magnitude. Put

$$
S_{\nu}=\sum_{i=1}^{N_{\nu}} c_{\nu i} \varphi_{\nu}\left(R_{\nu i} /\left(N_{\nu}+1\right)\right) \operatorname{sign} X_{\nu i}
$$

and extend the definition of $\varphi_{\nu}(u)$ to the whole interval $(0,1)$ according to (1.14). Furthermore, set

$$
\varphi(u)=-\left\{f^{\prime}\left(F^{-1}\left(\frac{1}{2}+\frac{1}{2} u\right)\right) / f\left(F^{-1}\left(\frac{1}{2}+\frac{1}{2} u\right)\right)\right\}
$$

and

$$
d_{\nu}^{2}=\sum_{i=1}^{N_{\nu}} c_{\nu i}^{2} \int_{-\infty}^{\infty}\left[f^{\prime}(x) / f(x)\right]^{2} f(x) d x
$$

Theorem 7.1. Let the conditions (1.3), (7.1), (7.2) and (7.3) be satisfied. Let (1.19) hold true for $\varphi_{\nu}$ used in (7.4) and $\varphi$ defined by (7.5).

Then the critical region $S_{\nu}>K_{\epsilon} d_{\nu}$, where $S_{\nu}, K_{\epsilon}$ and $d_{\nu}$ are given by (7.4), (1.11) and (7.6), respectively, provides an asymptotically uniformly most powerful test of $\beta=0$ against $\beta>0$ with limiting size $\epsilon$ and asymptotic power (1.20), where $d_{\nu}$ is given by (7.6).

Proof. The proof would be just the same as in Theorem 1.1. Usually the $c$ 's are constant, so that $S_{\nu}=2 S_{\nu}^{\prime}$-const, where

$$
S_{\nu}^{\prime}=\sum_{x_{\nu i}>0} \varphi_{\nu}\left(R_{\nu i} /\left(N_{\nu}+1\right)\right) .
$$

Special forms of $\varphi_{\nu}$ are obtained by setting $\varphi_{\nu}(u)=\varphi_{\nu}^{0}\left(\frac{1}{2}+\frac{1}{2} u\right), \varphi_{\nu}(u)=\varphi_{\nu}^{+}\left(\frac{1}{2}+\frac{1}{2} u\right)$ and $\varphi_{\nu}(u)=\varphi_{\nu}^{*}\left(\frac{1}{2}+\frac{1}{2} u\right)$, where $\varphi_{\nu}^{0}, \varphi_{\nu}^{+}$and $\varphi_{\nu}^{*}$ are given by $(2.1),(2.2)$ and (2.5), respestively. Upon taking $\varphi(u)=\operatorname{sign}\left(u-\frac{1}{2}\right)$, appearing in the first row of Table 1 , and putting $\varphi_{\nu}=\varphi_{\nu}^{*}\left(\frac{1}{2}+\frac{1}{2} u\right)$, we get the sign test, based on the statistic

$$
S_{\nu}^{\prime}=\sum_{X_{\nu i}>0} 1
$$

Upon taking $\varphi(u)=2 u-1$, we get the Wilcoxon test [14] for paired comparisons, based on the statistic

$$
\left(N_{\nu}+1\right) S_{\nu}^{\prime}=\sum_{x_{\nu i}>0} R_{\nu i} .
$$

8. A test for the uniform distribution. Let $f(x)$ be a density and set

$$
f_{\sigma}(x)=\sigma^{-1}(2 \pi)^{-\frac{1}{2}} \int_{-\infty}^{\infty} f(x-y) \exp \left(-\frac{1}{2}\left(y^{2} / \sigma^{2}\right)\right) d y
$$

It may be easily seen that $f_{\sigma}(x)$ satisfies condition (1.3) for any $f$ and $\sigma>0$. 
So we can take, for every $\sigma>0$, one of the statistics $S_{\nu}^{0}, S_{\nu}^{+}$and $S_{\nu}^{*}$, defined in Section 2, and observe what happens with the critical region when $\sigma \rightarrow 0$. At least in some cases, we obtain a limiting critical region, and may hope that it has some good asymptotic properties.

Let us illustrate this idea in the case of the uniform distribution over $(0,1)$. The probability density of its convolution with the normal probability density equals

$$
\begin{aligned}
f_{\sigma}(x) & =\sigma^{-1}(2 \pi)^{-\frac{1}{2}} \int_{x-1}^{x} \exp \left(-\frac{1}{2} t^{2} / \sigma^{2}\right) d t \\
& =\Phi\left(\frac{x}{\sigma}\right)-\Phi\left(\frac{x-1}{\sigma}\right) .
\end{aligned}
$$

Put $\varphi_{\sigma}(u)=-f_{\sigma}^{\prime}\left(F_{\sigma}^{-1}(u) / f_{\sigma}\left(F_{\sigma}^{-1}(u)\right)\right.$. Then

$$
\frac{\varphi_{\sigma}^{\prime}(u)}{\varphi_{\sigma}(u)}=-\frac{f_{\sigma}^{\prime \prime} f_{\sigma}-f_{\sigma}^{\prime 2}}{f_{\sigma}^{2} f_{\sigma}^{\prime}}=\left[f_{\sigma}^{\prime}-\frac{f_{\sigma}^{\prime \prime} f_{\sigma}}{f_{\sigma}^{\prime}}\right] f_{\sigma}^{-2}
$$

where, for fixed $u, 0<u<1, f(x) \rightarrow 1$ and $f_{\sigma}^{\prime} \rightarrow 0$, if $\sigma \rightarrow 0$. So

$$
\frac{\varphi_{\sigma}^{\prime}(u)}{\varphi_{\sigma}(u)} \sim-\frac{f_{\sigma}^{\prime \prime}}{f_{\sigma}^{\prime}}=\frac{\frac{1}{\sigma^{3}}\left[x e^{-\frac{1}{2} x^{2} / \sigma^{2}}-(x-1) e^{-\frac{1}{2}(x-1)^{2} / \sigma^{2}}\right]}{\frac{1}{\sigma}\left[e^{-\frac{1}{2} x^{2} / \sigma^{2}}-e^{-\frac{1}{2}(x-1)^{2} / \sigma^{2}}\right]} .
$$

This in conjunction with $u \sim x$ shows that

$$
\lim _{\sigma \rightarrow 0}\left|\varphi_{\sigma}^{\prime}(u) / \varphi_{\sigma}(u)\right|=\infty \quad 0<u<1, u \neq \frac{1}{2} .
$$

Now consider the statistic (2.6), where we drop the index $\nu$. From (8.2) if follows that the critical region generated by the statistic

$$
S_{\sigma}^{*}=\sum_{i=1}^{N}\left(c_{i}-\bar{c}\right) \varphi_{\sigma}\left(R_{i} /(N+1)\right)
$$

converges to one generated by the statistic

$$
W=0, I_{1} I_{2} \cdots I_{\left[\frac{1}{2} N\right]},
$$

where the right side represents the development of $W$ in the triadic system, $\left[\frac{1}{2} N\right]=\frac{1}{2} N$ for $N$ even and $\frac{1}{2}(N-1)$ for $N$ odd, and $I_{1}, \cdots I_{\left[\frac{1}{2} N\right]}$ are random variables defined as follows:

$$
\begin{aligned}
I_{k} & =2, & & \text { if } c_{D_{N-k}}>c_{D_{k}}, \\
& =1, & & \text { if } c_{D_{N-k}}=c_{D_{k}}, \\
& =0, & & \text { if } c_{D_{N-k}}<c_{D_{k}},
\end{aligned}
$$

where $D_{1}, \cdots, D_{N}$ is a random permutation of $(1, \cdots, N)$ defined by

$$
R_{D_{k}}=k,
$$

i.e. $D_{k}$ is the ordinal number of the observation whose rank (according to mag- 
nitude) is $k$. For example, if we have the following $c$ 's and $x$ 's,

\begin{tabular}{|c|c|c|c|c|c|}
\hline & 1 & 2 & 3 & 4 & 5 \\
\hline$x_{i}$ & 56 & 28 & -1 & 96 & 103 \\
\hline & 1 & 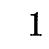 & 0 & 0 & 1 \\
\hline
\end{tabular}

then to the largest and smallest observation there corresponds $c_{5}=1$ and $c_{3}=0$, respectively, so that $c_{D_{6}}-c_{D_{1}}=c_{5}-c_{3}=1$, and $I_{1}=2$, etc. So we get $W=0.201$.

The test may be used for testing the hypothesis that the $X$ 's are uniformly distributed over $(a, b)$, where both $a$ and $b$ are unknown, against the alternative that $X_{i}$ is uniformly distributed over $\left(a-\beta c_{j}, b-\beta c_{i}\right)$, where $a, b$, are unknown and the $c$ 's are known.

Obviously, the limiting distribution of $W$ is not normal even under the hypothesis. Nevertheless the corresponding test might be asymptotically most powerful. The discussion of this problem would go however, beyond the scope of the present paper.

9. A universal asymptotically most powerful test. Now we shall construct a test, which is asymptotically most powerful for all densities $f(x)$ satisfying (1.3). However interesting this construction may be from the point of view of theory, it is of little use in practice because of slow convergence. First we generalize Theorem 1.1.

LEMMA 9.1. If the values of the functions $\varphi_{\nu}$ are random and under the hypothesis independent of the vectors $\left(X_{\nu 1}, \cdots, X_{\nu N_{\nu}}\right), 1 \leqq \nu<\infty$, and if condition (1.19) is replaced by

$$
\begin{aligned}
& \lim _{\nu \rightarrow \infty} P_{\nu}\left\{\int _ { 0 } ^ { 1 } \left[\varphi_{\nu}(u)-\right.\right.\left.(1 / \sigma) \varphi(u)]^{2} d u>\epsilon \mid \alpha=\alpha, \beta=0, \sigma=\sigma\right\} \\
&=0 \text { for every } \epsilon>0,-\infty<\alpha<\infty, \sigma>0,
\end{aligned}
$$

then Theorem 1.1 still holds.

Proof. Let $P_{\nu}\{\cdot\}$ and the conditional mean value $E_{\nu}\left[\cdot \mid \varphi_{\nu}\right]$ given $\varphi_{\nu}$ refer to some particular hypothesis $\alpha=\alpha, \beta=0, \sigma=\sigma$. Let $T_{\nu}$ and $T_{\nu}^{*}$ be given by (5.8) and (5.12). Then from (9.1) and from Theorem 3.1 of [6] it follows that for every $\epsilon>0$

$$
\lim _{\nu \rightarrow \infty} P_{\nu}\left\{E_{\nu}\left[\left(S_{\nu}-T_{\nu}^{*}\right)^{2} \mid \varphi_{\nu}\right]>\epsilon\right\}=0
$$

and

$$
\lim _{\nu \rightarrow \infty} P_{\nu}\left\{E_{\nu}\left[\left(T_{\nu}^{*}-T_{\nu}\right)^{2} \mid \varphi_{\nu}\right]>\epsilon\right\}=0 .
$$

Now, (9.2) and (9.3) imply (5.17). All other parts of the proof of Theorem 1.1 need no modification. The proof is finished.

REMARK 9.1. The function $(1 / \sigma) \varphi(u)$ corresponds to the density $f((x-\alpha) / \sigma)$ according to (1.9). 
Now we shall split the sample $\left(X_{\nu 1}, \cdots, X_{\nu N_{\nu}}\right)$ into two subsamples $\left(X_{\nu 1}, \cdots, X_{\nu K_{\nu}}\right)$ and $\left(X_{\nu K_{\nu}+1}, \cdots, X_{\nu N_{\nu}}\right)$, where $K_{\nu}$ is chosen so that

$$
\lim _{\nu \rightarrow \infty} K_{\nu}=\infty, \quad \lim _{\nu \rightarrow \infty}\left[K_{\nu} /\left(N_{\nu}-K_{\nu}\right)\right]=0,
$$

and

$$
\lim _{\nu \rightarrow \infty}\left\{\sum_{i=K_{\nu}+1}^{N_{\nu}}\left(c_{\nu i}-\overline{\bar{c}}_{\nu}\right)^{2} / \sum_{i=1}^{N_{\nu}}\left(c_{\nu i}-\bar{c}_{\nu}\right)^{2}\right\}=1,
$$

where

$$
\overline{\bar{c}}_{\nu}=\left(N_{\nu}-K_{\nu}\right)^{-1} \sum_{i=K_{\nu}+1}^{N_{\nu}} c_{\nu i} .
$$

This is always possible in view of (1.4). Condition $K_{\nu} \rightarrow \infty$ will enable us to find a $\varphi_{\nu}$ satisfying (9.1) and depending on $\left(X_{\nu 1}, \cdots, X_{\nu K_{\nu}}\right)$ only. Condition (9.5) shows, on the other hand, that the $S_{\nu}$-test based on the subsample $\left(X_{\nu K_{\nu}+1}, \cdots, X_{\nu N_{\nu}}\right)$ will have the same asymptotic power as the same test based on the whole sample $\left(X_{\nu 1}, \cdots, X_{\nu N_{\nu}}\right)$. So our problem will be solved by application of the $S_{\nu}$-test, with $\varphi_{\nu}$ determined from $\left(X_{\nu 1}, \cdots, X_{\nu K_{\nu}}\right)$, to $\left(X_{\nu K_{\nu}+1}, \cdots, X_{\nu N_{\nu}}\right)$. The first subsample $\left(X_{\nu 1}, \cdots, X_{\nu K_{\nu}}\right)$ will be small enough not to decrease the efficiency and large enough to get an estimator of $\varphi(u)$ which is consistent in the sense of (9.1).

LEMMA 9.2. Introduce numbers $m_{\nu}$,

$$
K_{\nu}^{\frac{4}{5}}<m_{\nu} \leqq K_{\nu}^{\frac{4}{5}}+1,
$$

and sequences $0=h_{\nu 0}<h_{\nu 1}<\cdots<h_{\nu n_{\nu}}<h_{\nu n_{\nu}+1}=K_{\nu}$ such that

$$
\lim _{\nu \rightarrow \infty} \max _{0 \leqq j \leqq n_{\nu}} \frac{\left|h_{\nu j+1}-h_{\nu j}\right|}{K_{\nu}^{\frac{5}{j}}}=\lim _{\nu \rightarrow \infty} \min _{0 \leqq j \leqq n_{\nu}} \frac{\left|h_{\nu j+1}-h_{\nu j}\right|}{K_{\nu}^{\frac{5}{j}}}=1,
$$

and set, for $1 \leqq j \leqq n_{\nu}$,

$$
k_{\nu j}=h_{\nu j}+m_{\nu}, \quad l_{\nu j}=h_{\nu j}-m_{\nu} .
$$

Let $V_{\nu 1}<\cdots<V_{\nu K_{\nu}}$ be the observations $X_{\nu 1}, \cdots, X_{\nu K_{\nu}}$ rearranged according to ascending magnitude. Suppose that (9.4) is satisfied. Finally, for $h_{\nu j} / K_{\nu}<$ $i /\left(N_{\nu}-K_{\nu}+1\right) \leqq h_{\nu j+1} / K_{\nu}, 1 \leqq j<n_{\nu}$, put

(9.10) $\varphi_{\nu}\left[i /\left(N_{\nu}-K_{\nu}+1\right)\right]=\frac{1}{2} K_{\nu}^{-\frac{3}{30}}\left[\left(V_{k_{\nu j}}-V_{l_{\nu j}}\right)^{-1}-\left(V_{k_{\nu j+1}}-V_{l_{\nu j+1}}\right)^{-1}\right]$, and set $\varphi_{\nu}\left[i /\left(N_{\nu}-K_{\nu}+1\right)\right]=0$ otherwise. Complete the definition of $\varphi(u)$ so as to be constant over intervals $\left[i /\left(N_{\nu}-K_{\nu}\right),\left((i+1) /\left(N_{\nu}-K_{\nu}\right)\right)\right]$, $0 \leqq i<N_{\nu}-K_{\nu}$. Then (9.1) holds true for any density satisfying (1.3).

Proof. Obviously, it suffices to prove (9.1) for $\alpha=0, \sigma=1$. Then the random variables $U_{\nu i}=F\left(X_{\nu i}\right), 1 \leqq i \leqq K_{\nu}$, will be uniformly distributed over $(0,1)$ and $Z_{\nu l}<\cdots<Z_{\nu k_{\nu}}$, where $Z_{\nu i}=F\left(V_{\nu i}\right)$, will be the ordered sample from the uniform distribution. We know that

$$
E Z_{\nu i}=i /\left(K_{\nu}+1\right),
$$


and

$$
\operatorname{Var} Z_{\nu i}=\left(i\left(K_{\nu}-i+1\right) /\left(K_{\nu}+1\right)^{2}\left(K_{\nu}+2\right)\right)<\left(1 / K_{\nu}\right) .
$$

Consequently, by the Tchebyshev inequality, the probability of the event $A_{\nu}$,

$$
\begin{aligned}
A_{\nu}=\bigcup_{j=1}^{n_{\nu}}\left\{\left|Z_{\nu k_{\nu j}}-\left[k_{\nu j} /\left(K_{\nu}+1\right)\right]\right|>\left(m_{\nu} / K_{\nu}\right) K_{\nu}^{-\frac{1}{5}}\right\} \\
\cup \bigcup_{j=1}^{n_{\nu}}\left\{\left|Z_{\nu 1_{\nu j}}-\left[l_{\nu j} /\left(K_{\nu}+1\right)\right]\right|>\left(m_{\nu} / K_{\nu}\right) K_{\nu}^{-\frac{1}{5}}\right\}
\end{aligned}
$$

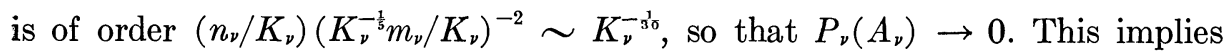
that for every $\epsilon>0$

(9.14) $\lim _{\nu \rightarrow \infty} P_{\nu}\left\{\max _{i \leqq j \leqq n_{\nu}} K_{\nu}^{-3^{\prime} \frac{1}{\sigma}}\left|\left[Z_{\nu k_{\nu j}}-Z_{\nu l_{\nu j}}\right]^{-1}-\frac{1}{2}\left(K_{\nu} / m_{\nu}\right)\right|>\epsilon\right\}=0$.

On the other hand, from $Z_{\nu i}=F\left(V_{\nu i}\right)$ it follows that

$$
Z_{\nu k_{\nu j}}-Z_{\nu l_{\nu j}}=\left(V_{\nu k_{\nu j}}-V_{\nu l_{\nu j}}\right) f\left(B_{\nu j}\right)
$$

where

$$
V_{\nu l_{\nu j}} \leqq B_{\nu j} \leqq V_{\nu k_{\nu j}}
$$

Hence (9.10) may be rewritten as follows:

$$
\varphi_{\nu}\left(\frac{i}{N_{\nu}-K_{\nu}+1}\right)=\frac{1}{2} K_{\nu}^{-3^{1} \delta}\left[\frac{f\left(B_{\nu j}\right)}{Z_{\nu k_{\nu j}}-Z_{\nu l_{\nu j}}}-\frac{f\left(B_{\nu j+1}\right)}{Z_{\nu k_{\nu j+1}}-Z_{\nu l_{\nu j+1}}}\right] .
$$

Now from boundedness of $f(x)$ (implied by (1.3)) and from (9.14) it follows that for every $\epsilon>0$

$$
\begin{aligned}
\lim _{\nu \rightarrow \infty} P_{\nu}\left\{\max _{h_{\nu 1} / K_{\nu}<i /\left(N_{\nu}-K_{\nu}+1\right) \leqq h_{\nu n_{\nu}} / K_{\nu}} \mid \varphi\left[i /\left(N_{\nu}-K_{\nu}+1\right)\right]\right. \\
\left.-K_{\nu}^{\frac{1}{6}}\left[f\left(B_{\nu j}\right)-f\left(B_{\nu j+1}\right)\right] \mid>\epsilon\right\}=0 .
\end{aligned}
$$

Moreover, from (9.7), (9.8), (9.9), (9.16) and $P_{\nu}\left(A_{\nu}\right) \rightarrow 0, A_{\nu}$ given by (9.13), it follows that for every $\epsilon>0$

(9.19) $\lim _{\nu \rightarrow \infty} P_{\nu}\left\{\max _{l \leqq j \leqq n_{\nu}}\left|\left[K_{\nu}^{\frac{1}{b}} /\left(F\left(B_{\nu j+1}\right)-F\left(B_{\nu j}\right)\right)\right]-1\right|>\epsilon\right\}=0$

The relations (9.18) and (9.19) imply that it suffices to prove (9.1) for the step function defined by

$$
\begin{gathered}
\bar{\varphi}_{\nu}\left(i /\left(N_{\nu}-K_{\nu}+1\right)\right)=\left\{\left[f\left(B_{\nu j}\right)-f\left(B_{\nu j+1}\right)\right] /\left[F\left(B_{\nu j+1}\right)-F\left(B_{\nu j}\right)\right]\right\} \\
\text { if }\left(h_{\nu j} / K_{\nu}\right)<\left(i /\left(N_{\nu}-K_{\nu}+1\right)\right) \leqq\left(h_{\nu j+1} / K_{\nu}\right), \quad 1 \leqq j<n_{\nu}, \\
=0 \text { otherwise }
\end{gathered}
$$

and by the requirement to be constant over intervals $\left(i /\left(N_{\nu}-K_{\nu}\right),(i+1) /\right.$ $\left.\left(N_{\nu}-K_{\nu}\right)\right), 0 \leqq i<N_{\nu}-K_{\nu}$. Put $C_{\nu j}=F\left(B_{\nu j}\right), 1 \leqq j \leqq n_{\nu}$, and observe that

$$
\frac{f\left(B_{\nu j}\right)-f\left(B_{\nu j+1}\right)}{F\left(B_{\nu j+1}\right)-F\left(B_{\nu j}\right)}=\frac{\int_{C_{\nu j}}^{C_{\nu j+1}} \varphi(u) d u}{C_{\nu j+1}-C_{\nu j}},
$$


and, on account of (9.16),

$$
Z_{\nu l_{\nu j}} \leqq C_{\nu j} \leqq Z_{\nu k_{\nu j}}, \quad 1 \leqq j \leqq n_{\nu}
$$

In view of $P_{\nu}\left(A_{\nu}\right) \rightarrow 0, A_{\nu}$ given by (9.13), we have

$$
\lim _{\nu \rightarrow \infty} P_{\nu}\left\{\max _{1 \leqq j \leqq n_{\nu}}\left|C_{\nu j}-\left(h_{\nu j} / K_{\nu}\right)\right|>4\left(m_{\nu} / K_{\nu}\right)\right\}=0,
$$

and, on account of $n_{\nu} m_{\nu} / K_{\nu} \rightarrow 0$, for every $\epsilon>0$,

$$
\lim _{\nu \rightarrow \infty} P_{\nu}\left\{\sum_{j=1}^{n_{\nu}}\left|C_{\nu j}-\left(h_{\nu j} / K_{\nu}\right)\right|>\epsilon\right\}=0 .
$$

Introduce another step function $\bar{\varphi}_{\nu}$,

$$
\begin{aligned}
\bar{\varphi}_{\nu}(u) & =\frac{f\left(B_{v j}\right)-f\left(B_{v j+1}\right)}{F\left(B_{v j+1}\right)-F\left(B_{v j}\right)} \text { if } \quad C_{\nu j}<u \leqq C_{\nu j+1}, \quad 1 \leqq j<n_{\nu} . \\
& =0 \quad \text { otherwise. }
\end{aligned}
$$

Now, because $\varphi(u)$ is quadratically integrable, to any $\epsilon>0$ there exists a $\delta>0$ such that

$$
\max _{0 \leqq j \leqq n_{\nu}}\left|C_{\nu j}-C_{\nu j+1}\right|<\delta, \quad C_{\nu 0}=0, C_{\nu_{n+1}}=1
$$

entails

$$
\int_{0}^{1}\left[\overline{\bar{\varphi}}_{\nu}(u)-\varphi(u)\right]^{2} d u<\epsilon .
$$

(We remind the reader of relation (9.21).) Now (9.26) is, obviously, fulfilled for every $\delta>0$ with limiting probability 1 , and, consequently, for every $\epsilon>0$,

$$
\lim _{\nu \rightarrow \infty} P_{\nu}\left\{\int_{0}^{1}\left[\overline{\bar{\varphi}}_{\nu}(u)-\varphi(u)\right]^{2} d u>\epsilon\right\}=0 .
$$

On the other hand, from the very definitions of $\bar{\varphi}_{\nu}$ and $\bar{\varphi}_{\nu}$, it follows that

$$
\int_{0}^{1}\left[\bar{\varphi}_{\nu}(u)-\bar{\varphi}_{\nu}(u)\right]^{2} d u<2 \int_{\Lambda_{\nu}} \bar{\varphi}_{\nu}^{2}(u) d u
$$

where the Lebesgue measure of $\Lambda_{\nu}$ tends to 0 in $P_{\nu}$-probability in view of $K_{\nu} /\left(N_{\nu}-K_{\nu}\right) \rightarrow 0$ and (9.24). This, in connection with uniform integrability of functions $\bar{\varphi}_{\nu}^{2}$ corresponding to all possible divisions $\left[C_{\nu 1}, \cdots, C_{\nu n_{\nu}}\right]$, and on account of (9.28), implies, for every $\epsilon>0$,

$$
\lim _{\nu \rightarrow \infty} P_{\nu}\left\{\int_{0}^{1}\left[\bar{\varphi}_{\nu}(u)-\varphi(u)\right]^{2} d u>\epsilon\right\}=0 .
$$

The proof is terminated.

THeorem 9.1. Let assumptions (1.3), (1.4) and (1.5) be satisfied. Let $K_{\nu}$ satisfy. the conditions (9.4) and (9.5). Let $V_{\nu 1}<\cdots<V_{\nu K_{\nu}}$ be the ordered subsample $\left(X_{\nu 1}, \cdots, X_{\nu K_{\nu}}\right)$ and let $R_{\nu i}$ denote the rank of $X_{\nu i}$ in the subsample $\left(X_{\nu K_{\nu}+1}, \cdots\right.$, 
$\left.X_{\nu N_{\nu}}\right), K_{\nu}<i \leqq N_{\nu}, 1 \leqq \nu<\infty$. Let $\varphi_{\nu}$ be defined by (9.10) and $\overline{\bar{c}}_{\nu}$ by (9.6). Then the critical region

$$
\begin{aligned}
\sum_{i=K_{\nu}+1}^{N_{\nu}}\left(c_{\nu i}-\overline{\bar{c}}_{\nu}\right) \varphi_{\nu}\left(R_{\nu i} /\left(N_{\nu}-\right.\right. & \left.\left.K_{\nu}+1\right)\right) \\
& >K_{\epsilon}\left[\sum_{i=K_{\nu}+1}^{N_{\nu}}\left(c_{\nu i}-\overline{\bar{c}}_{\nu}\right)^{2} \int_{0}^{1} \varphi_{\nu}^{2}(u) d u\right]^{\frac{1}{2}}
\end{aligned}
$$

provides an asymptotically uniformly most powerful test of $\beta=0$ against $\beta>0$ with limiting size $\epsilon$ and asymptotic power (1.20) for all densities in question.

Proof. The proof follows immediately from Lemmas 9.1 and 9.2.

\section{REFERENCES}

[1] CAPON, JACK (1961). Asymptotic efficiency of certain locally most powerful rank tests. Ann. Math. Statist. 32 88-100.

[2] Chernoff, H. and Savage, I. R. (1958). Asymptotic normality and efficiency of certain nonparametric tests statistics. Ann. Math. Statist. 29 972-994.

[3] Dwass, M. (1956). The large sample power of rank order tests in the two sample case. Ann. Math. Statist. 27 352-374.

[4] Fraser, D. A. S. (1956). Nonparametric Methods in Statistics. John Wiley and Sons, New York.

[5] Gnedenko, B. V. and Kolmogorov, A. N. (1954). Limit Distributions for Sums of Independent Random Variables. Addison-Wesley, Cambridge.

[6] HÁJeK, J. (1961). Some extensions of the Wald-Wolfowitz-Noether theorem. Ann. Math. Statist. 32 506-523.

[7] Ibragimov, J. A. (1956). On the composition of unimodal distributions. Teoriya Veroyatnostey I 283-288.

[8] LeCAM, L. (1960). Locally asymptotically normal families of distributions. Univ. of Calif. Pubs. in Stat. 3 37-98.

[9] LeCAM, L. (196-). Asymptotic normality. Independent identically distributed case. Unpublished manuscript.

[10] Mikulski, P. W. (1961). Some problems in the asymptotic theory of testing statistical hypotheses (efficiency of non-parametric procedures). Ph.D. Thesis, University of California, Graduate Division, Northern Section.

[11] Neyman, J. (1958). Optimal asymptotic tests of composite statistical hypotheses. The H. Cramér Jubilee Volume, Almquist and Wiksell, Uppsala.

[12] Savage, I. R. (1956). Contributions to the theory of rank order statistics-the two sample case. Ann. Math. Statist. 27 590-615.

[12a] Stein, Charles (1956). Efficient nonparametric testing and estimation. Third Berkeley Symp. Math Statist. Prob. 187-195. Univ. of California Press.

[13] van der Waerden, B. L. (1957). Mathematische Statistik. Springer-Verlag, BerlinGöttingen-Heidelberg.

[14] Wilcoxon, F. (1945). Individual comparisons by ranking methods. Biometrics $180-83$. 